\title{
Inhibition of cancer cells using target-specific 2A3 antibody-conjugated gold nanoclusters
}

\section{Jui-Chi Kuo ${ }^{1} \mathbb{D}$, Tsung-Rong Kuo ${ }^{1,2} \mathbb{D}$, Fajar Rinawati ${ }^{3} \mathbb{D}$, Erna Susilowati ${ }^{3} \mathbb{D}$, Sucipto ${ }^{3} \mathbb{D}$, Dyah Ika Krisnawati ${ }^{3^{*}}$ (D)}

'Graduate Institute of Nanomedicine and Medical Engineering, College of Biomedical Engineering, Taipei Medical University, Taipei 11031, Taiwan

${ }^{2}$ International Ph.D. Program in Biomedical Engineering, College of Biomedical Engineering, Taipei Medical University, Taipei 11031, Taiwan

${ }^{3}$ Dharma Husada Nursing Academy, Kediri 64114, Indonesia

${ }^{*}$ Correspoding author: Jl. Penanggungan No.41a, Bandar Lor, Kec. Kota Kediri, Kota Kediri, Jawa Timur 64114, Indonesia. Email: dyahkrisna77@gmail.com

\begin{abstract}
Background: Metal nanoclusters (NCs) with outstanding structural and optical properties have been intensively validated for applications in nanomedicine and nanotechnology. Carcinoembryonic antigen-related cell adhesion molecule 6 (CEACAM6) is overexpressed in many cancer cells.

Objective: The gold nanoclusters conjugated with a single domain antibody targeting CEACAM6 of 2A3 (2A3AuNCs) were synthesized for the inhibition of cancer cells.

Methods: 2A3-AuNCs were prepared via a facile hydrothermal approach. The cell viability was measured by resazurin dye reduction assay. The cell death was analyzed by fluorescence imaging.

Results: Structural and optical characterizations demonstrated the successful synthesis of 2A3-AuNCs with a roughly spherical shape and a size of $2.35 \mathrm{~nm}$. The 2A3-AuNCs revealed a maximum fluorescence intensity at $350 \mathrm{~nm}$ with a fluorescence quantum yield of $4.0 \%$. The cell viability assay indicated that 2A3-AuNCs could inhibit the growths of cancer cells with overexpressed CEACAM6, including breast cancer MDA-MB-231 and MDA-MB-468 cells. The fluorescence imaging results also demonstrated that 2A3-AuNCs could inhibit the growth of cancer cells with MDA-MB-231 and MDA-MB-468 cells.

Conclusion: Combination with the results of cell viability assay and fluorescence imaging, the surface ligand of $2 \mathrm{~A} 3$ antibody on 2A3-AuNCs exhibited promising inhibition of CEACAM6 overexpressed cancer cells. Our work provides a potential application of AuNCs in cancer therapy.
\end{abstract}

Keywords: 2A3, CEACAM6, gold nanoclusters, inhibition, therapy

\section{Introduction}

Recent advancements of nanomaterials have been focused on applications in nanomedicine and nanotechnology based on their outstanding chemical and physical properties [1-14]. Among these nanomaterials, metal nanoclusters (NCs) composed of several to hundreds of metal atoms with unique structural and optical characteristics have been extensively demonstrated for the research fields in imaging, detection, and therapy [15-17]. Great efforts have been made to prepare fluorescent metal NCs using various surface ligands, including small molecules, polymers, and biomacromolecules [18]. For example, gold nanoclusters (AuNCs) conjugated with the surface ligand of glucose have been demonstrated as a target-specific fluorescent probe to analyze the glucose metabolism in the glucose transporter overexpressed brain cancer cells [19]. Fluorescent AuNCs and silver nanoclusters (AgNCs) with surface modification of reactive oxygen species (ROS) scavenger of cysteine have been exploited as a highly biocompatible probe for confocal microscopy [20]. The thiol-modified liquid crystal of 4'-(2-mercaptoethyl)-(1,1'-biphenyl)-4- 
carbonitrile has been applied as a surface ligand to prepare fluorescent AuNCs [21]. Fluorescent copper nanoclusters (CuNCs) modified with DNA have been utilized for sensitively fluorimetric detection of the mismatch type in a specific DNA sequence for the diagnosis and risk assessment of cancer in the early stage[22]. These metal nanoclusters with different surface modifications, including amino acids, polymers, peptides, antibodies, DNA, and so forth, have revealed outstanding biocompatibility, excellent photostability, and high water solubility for biomedical applications in theranostics.

Various metal NCs have been demonstrated as promising antibacterial agents due to their ultrasmall sizes to increase interaction with bacteria [23-25]. For example, AuNCs conjugated with 6-mercaptohexanoic acid (MHA) have shown higher antibacterial activity in comparison with MHA conjugated gold nanoparticles and the complexes of $\mathrm{Au}(\mathrm{I})-\mathrm{MHA}$ against Gram-negative Escherichia coli (E. coli) and Gram-positive Staphylococcus aureus (S. aureus) [26]. The amino acid of cysteine-conjugated AuNCs have exhibited antibacterial activity because of the significant increase of intracellular ROS induced by cysteine-conjugated AuNCs in bacteria [27]. The bacitracin-conjugated AuNCs. AgNCs and CuNCs have been validated the antibacterial activities because of the destruction of the bacterial cell wall and the increase of intracellular ROS generation [28]. Real-time observation has demonstrated the antibacterial mechanism between the surface ligand of glutathione conjugated AuNCs and bacteria by in situ liquid cell transmission electron microscopy (TEM) [29]. Although the intensive achievements studies have proven the antibacterial activity of metal NCs, there are only very few studies to demonstrate the cancer therapy using metal NCs.

The investigation of cancer therapy is an urgent task for biomedical applications based on metal nanoclusters. Herein, AuNCs conjugated with the surface ligand of antibody were developed to demonstrate their application in cancer therapy. Carcinoembryonic antigen-related cell adhesion molecule 6 (CEACAM6) is overexpressed in many cancer cells [30]. In this work, a single domain antibody targeting CEACAM6, 2A3, was utilized as a surface ligand to prepare $2 \mathrm{~A} 3$ conjugated AuNCs (2A3-AuNCs). Structural and optical properties were characterized by TEM, ultraviolet-visible (UVVis) spectroscopy, and fluorescence spectroscopy. The cytotoxicities of 2A3-AuNCs incubated with Vero, MDA-MB-231, and MDA-MB-468 cells were respectively examined by resazurin dye reduction assay. To investigate the death of cells, 2A3AuNCs incubated with Vero, MDA-MB-231, and MDA-MB-468 cells were separately measured by fluorescence images.

\section{Methods}

Preparation of $2 \mathrm{~A} 3$ conjugated gold nanoclusters

The 2A3 antibody was synthesized according to previous literature [30]. In this work, 2A3AuNCs were synthesized by a facile hydrothermal approach. For preparing 2A3-AuNCs, $1 \mathrm{~mL}$ of $\mathrm{HAuCl}_{4}$ aqueous solution was first added to a sample vial. Then $2 \mathrm{~mL}$ of $2 \mathrm{~A} 3$ antibody solution was added to the sample vial with $\mathrm{HAuCl}_{4}$ aqueous solution under vigorous stirring. Afterward, $3 \mathrm{~m}$ $\mu \mathrm{L}$ of $1 \mathrm{mM} \mathrm{NaOH}$ aqueous solution was added to the sample vial. After stirring for one week in the dark, the solution containing 2A3-AuNCs was obtained. For purifying step, the solution of 2A3-AuNCs was centrifuged at $15000 \mathrm{rpm}$ at $4{ }^{\circ} \mathrm{C}$ for $10 \mathrm{~min}$. The supernatant solution containing 2A3-AuNCs was then stored at $4{ }^{\circ} \mathrm{C}$ for the following experiments.

\section{Cell viability assay of $2 \mathrm{A3}-\mathrm{AuNCs}$}

Kidney epithelial Vero cells, breast cancer MDAMB-231 cells, and breast cancer MDA-MB-468 cells were cultured in Dulbecco's modified Eagle medium (DMEM) with 10\% (v/v) fetal bovine serum (FBS). For cell culture, Vero, MDA-MB-231, and MDA-MB-468 cells were cultured in 96-well plates for $24 \mathrm{~h}$ and then washed twice with a phosphate-buffered saline (PBS) solution. Afterward, Vero, MDA-MB-231, and MDA-MB-468 cells in 96-well plates were respectively incubated with 2A3-AuNC solutions $(270,135,67.5,33.75,16.88$, 8.44, 4.22, 2.11, and $1.05 \mu \mathrm{g} / \mathrm{mL}$ ). The sterilized water was used for the control experiments. The 

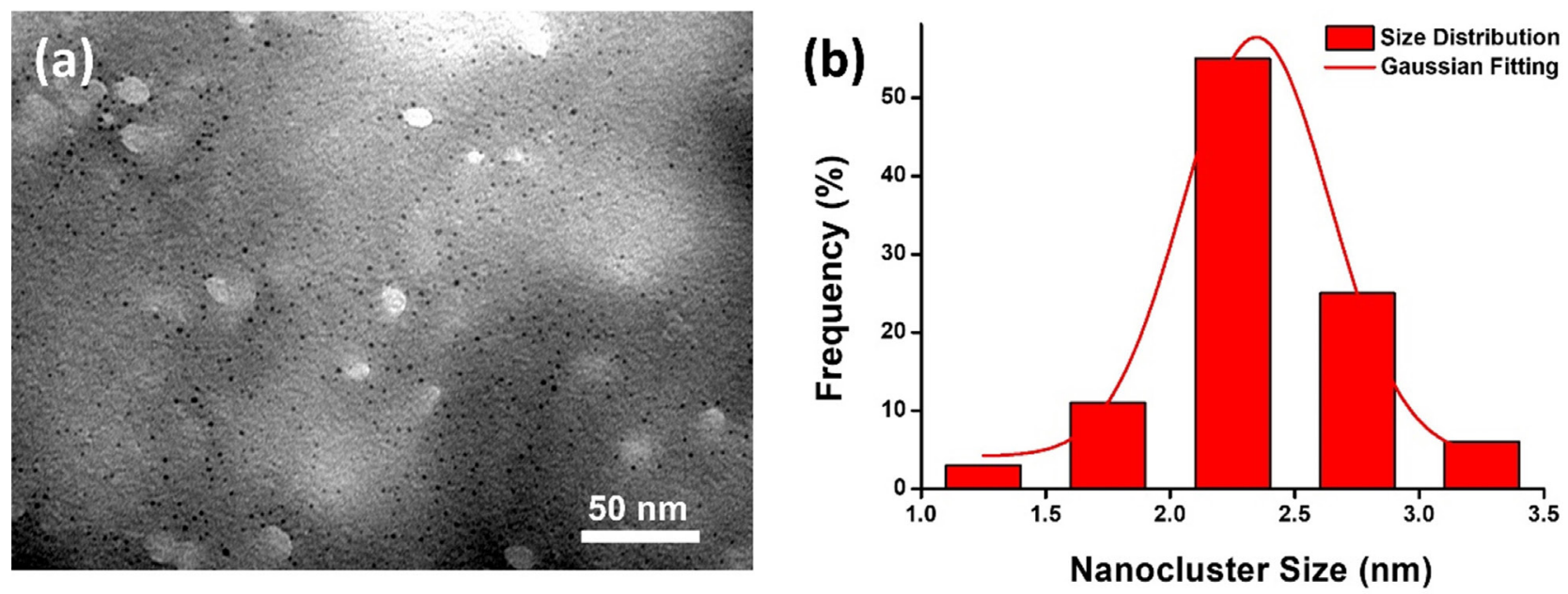

Figure 1. Structural characterizations of 2A3-AuNCs. (a) TEM image of 2A3-AuNCs and (b) histogram of the nanocluster size distribution of 2A3-AuNCs and its Gaussian fitting curves.

cell viabilities of Vero, MDA-MB-231, and MDAMB-468 cells incubated with 2A3-AuNCs were measured by resazurin dye reduction assay. After incubating 2A3-AuNCs with Vero, MDA-MB-231, and MDA-MB-468 cells for $24 \mathrm{~h}$, the resazurin (final concentration of $0.02 \mathrm{mg} / \mathrm{mL}$ ) was added to each well of the 96-well plates and then incubated for $4 \mathrm{~h}$. The absorbances at 570 and $600 \mathrm{~nm}$ were measured by a plate reader. The cell viability assay was replicated eight times for each cell line.

\section{Analysis of cell death by fluorescence imaging}

SYTOX green nucleic acid stain was applied to stain dead cells by fluorescence imaging. Herein, SYTOX green nucleic acid stain $(5 \mu \mathrm{M})$ was added to cells and then the cells, and SYTOX green nucleic acid stain were incubated in a shaker at $200 \mathrm{rpm}$ and $37{ }^{\circ} \mathrm{C}$ for $15 \mathrm{~min}$ in the dark. Afterward, the solution of SYTOX green nucleic acid stain was carefully removed and the cells were further washed by medium for three times. Sequentially, Hoechst 33342 nucleic acid stain $(1 \mu \mathrm{g} / \mathrm{mL}$ ) was added to stain total cells. After incubation of cells and Hoechst 33342 nucleic acid stain in a shaker at $200 \mathrm{rpm}$ and $37{ }^{\circ} \mathrm{C}$ for $10 \mathrm{~min}$ in dark, the solution of Hoechst 33342 nucleic acid stain was carefully removed and the cells were further washed by medium for three times. The cells stained by SYTOX green nucleic acid stain and Hoechst 33342 nucleic acid stain were observed using microscope (Leica DM1000).
To obtain better image contrast, we assigned a false green for the fluorescence of SYTOX stain channel and a false blue for the Hoechst 33342 stain channel.

\section{Results}

\section{Structural characterizations of 2A3-AuNCs}

TEM (Hitachi HT-7700) was applied to examine the shape of 2A3-AuNCs. As shown in the TEM image of Figure $1 \mathrm{a}$, the shape of $2 \mathrm{~A} 3$-AuNCs exhibited a roughly spherical shape. Furthermore, a histogram was systematically calculated the size distribution of 2A3-AuNCs based on 100 nanoclusters in the TEM image of Figure 1a as shown in Figure 1b. A Gaussian fitting curve of Figure $1 \mathrm{~b}$ was simulated, and the result of the Gaussian fitting curve indicated that the average size of 2A3-AuNCs was calculated to be $2.35 \mathrm{~nm}$.

\section{Optical properties of 2A3-AuNCs}

UV-Vis absorption spectrometer (Jasco V-770) and fluorescence spectrometer (Jasco FP-8500) were applied to further examine optical properties to characterize 2A3-AuNCs. As shown in Figure 2a, the absorption spectrum of 2A3-AuNCs showed no surface plasmon absorption. The disappearance of the surface plasmon absorption of 2A3-AuNCs can be attributed to that 2A3-AuNCs exhibited high gold oxidation states to result in a lack of free electrons for the generation of coherent 

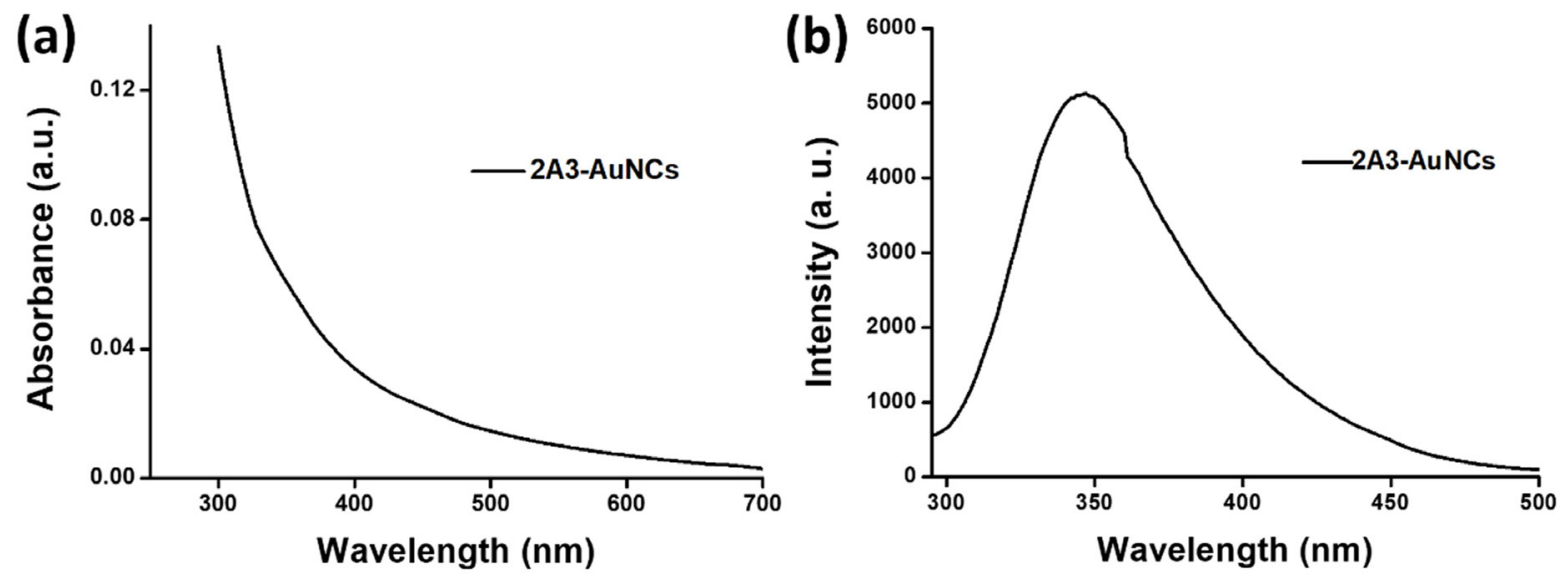

Figure 2. Optical characterizations of 2A3-AuNCs. (a) UV-Vis absorption spectrum of 2A3-AuNCs and (b) Fluorescence spectrum of 2A3-AuNCs.

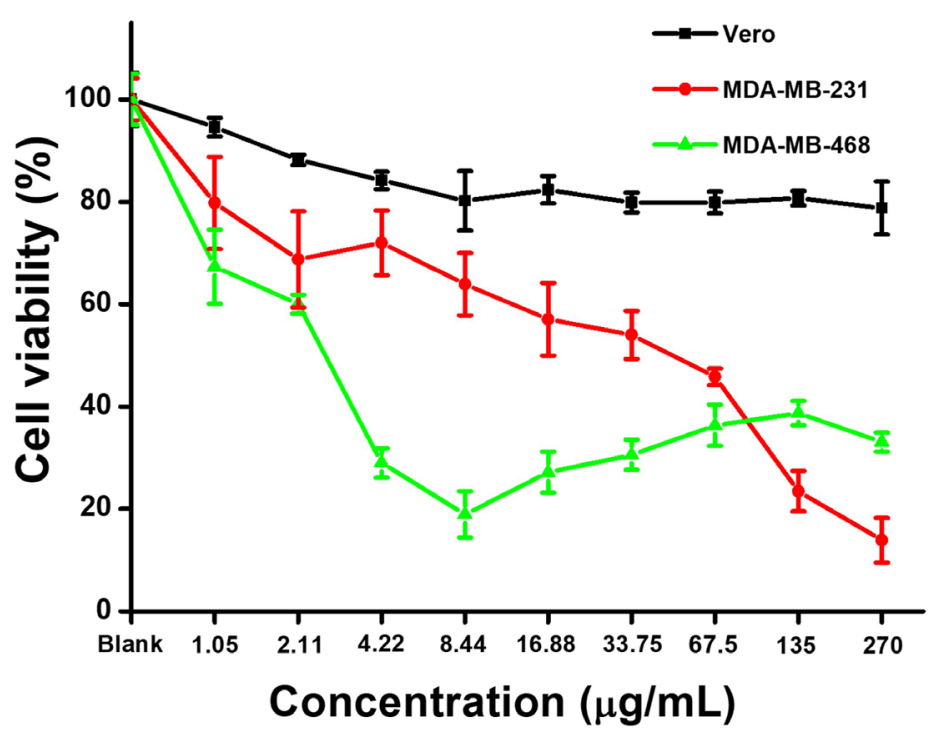

Figure 3. Cell viability assay of $2 A 3-A u N C s$. Cell viabilities by the resazurin dye reduction assay with the concentration range from 1.05 to $270 \mu \mathrm{g} / \mathrm{mL}$ of $2 A 3-A u N C s$ in Vero, MDA-MB-231, and MDA-MB-468 cells. The sterilized water was used in the control experiments.

oscillations [31]. Moreover, the fluorescence spectrum of 2A3-AuNCs revealed a maximum fluorescence intensity at $350 \mathrm{~nm}$, as shown in Figure $2 \mathrm{~b}$. The fluorescence quantum yield of 2A3-AuNCs was $4.0 \%$ by integrating the sphere (Jasco ILF-835). The fluorescence of 2A3-AuNCs can be ascribed to the fluorescent mechanism of aggregation-induced emission (AIE). Several reports have proven that the AIE fluorescence of AuNCs is caused by the aggregation of $\mathrm{Au}(\mathrm{I})$ and ligand on the surface of AuNCs [32-34]. Overall, structural and optical characterizations of 2A3AuNCs confirmed that the facile hydrothermal approach successfully synthesized fluorescent 2A3-AuNCs.

\section{Cell viability of 2A3-AuNCs}

To examine the potential for cancer therapy, the cell viabilities of 2A3-AuNCs were respectively evaluated in Vero, MDA-MB-231, and MDAMB-468 cells. The water-soluble 2A3-AuNCs with different concentrations $(270,135,67.5,33.75$, 16.88, 8.44, 4.22, 2.11, and $1.05 \mu \mathrm{g} / \mathrm{mL}$ ) were prepared to investigate their cytotoxicities. As shown in Figure 3, the resazurin dye reduction assay revealed high cell viabilities ( $>80 \%$ ) for 2A3-AuNCs. However, for MDA-MB-231 and MDAMB-468 cells with overexpressed CEACAM6, the cell viabilities decreased with the concentration of 2A3-AuNCs. The results of cell viabilities of 2A3-AuNCs indicated that antibody of $2 \mathrm{~A} 3$ on the 


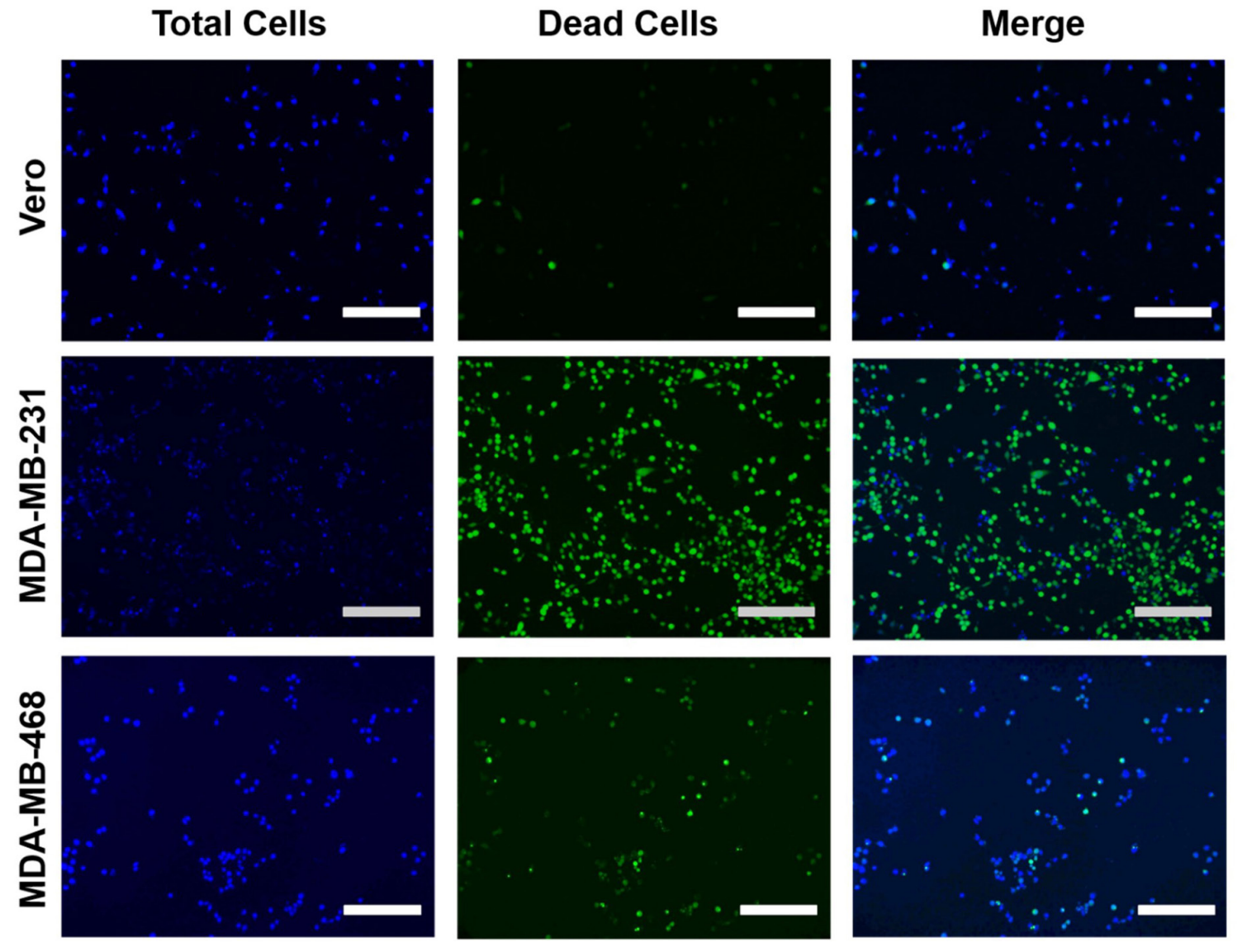

Figure 4. Cell death assay of 2A3-AuNCs. Fluorescence images of 2A3-AuNCs incubated with Vero, MDA-MB-231, and MDAMB-468 cells for $120 \mathrm{~min}$. The blue and green pseudocolors represent the fluorescent signals of total cells (stained with Hoechst 33342) and dead cells (stained with SYTOX green), respectively. The scale bars were $1 \mathrm{~mm}$.

surface of 2A3-AuNCs could target onto CEACAM6 overexpressed MDA-MB-231 and MDA-MB-468 cells and then inhibit the growth of MDA-MB-231 and MDA-MB-468 cells.

\section{Investigation of cell death induced by 2A3- AuNCs}

To investigate the cell death, fluorescence images of cells including Vero, MDA-MB-231, and MDA-MB-468 cells were respectively examined after incubation with 2A3-AuNCs for $120 \mathrm{~min}$. As shown in fluorescence images of Figure 4, the total numbers of Vero, MDA-MB-231, and MDAMB-468 cells revealed no significant difference. Moreover, after incubation with 2A3-AuNCs, no significant death of Vero cells was observed in the fluorescence image. However, for MDA-MB-231 and MDA-MB-468 cells, drastic cell deaths were observed after incubation with 2A3-AuNCs. The results of fluorescence images indicated that the ligand of $2 \mathrm{~A} 3$ on the surface of 2A3-AuNCs could inhibit the growths of cancer cells with CEACAM6 overexpressed MDA-MB-231 and MDAMB-468 cells.

\section{Discussion}

The treatment of cancer is an urgent issue for biomedical applications based on metal nanoclusters. Herein, the 2A3-AuNCs were synthesized to inhibit cancer cells by the facile hydrothermal approach. The structural and optical properties of 2A3-AuNCs confirmed the successful preparation of fluorescent 2A3-AuNCs with the roughly spherical shape and the size of $2.35 \mathrm{~nm}$ by the facile hydrothermal approach. The cell viability assay demonstrated that 2A3AuNCs could inhibit the growths of cancer cells, including MDA-MB-231 and MDA-MB-468 breast cells. The fluorescence images also indicated that 2A3-AuNCs could inhibit the growths of 
cancer cells of MDA-MB-231 and MDA-MB-468 breast cells. Based on the similar results of cell viability assay and fluorescence imaging, the surface ligand of $2 \mathrm{~A} 3$ antibody on 2A3-AuNCs exhibited significant inhibition of cancer cells with CEACAM6 overexpressed, including MDA-MB-231 and MDA-MB-468 breast cells. For further clinical application, the in vivo studies of 2A3-AuNCs are still needed in the future.

\section{Conclusion}

In conclusion, fluorescent 2A3-AuNCs were successfully prepared by the facile hydrothermal approach. The optical and structural characterizations of 2A3-AuNCs were demonstrated by TEM image and UV-Vis spectrum. The results of the cell viability assay confirmed that 2A3-AuNCs revealed cancer cell inhibition for CEACAM6 overexpressed MDAMB-231 and MDA-MB-468 cells. The results of fluorescence imaging also indicated that the $2 \mathrm{~A} 3$ antibody on the surface of 2A3-AuNCs showed significant cancer cell inhibition for CEACAM6 overexpressed MDA-MB-231 and MDA-MB-468 cells. Overall, our studies showed that 2A3-AuNCs could be a promising fluorescent probe for the detection and therapy in the cancer cell.

\section{Acknowledgment}

We would like to thank Dr. Chi-Ming Lee and Chun-Chih Liu for their help with materials characterizations at the TMU Core Facility Center.

\section{Funding}

We acknowledge the Ministry of Science and Technology, Taiwan (grant no.: MOST 109-2113-M-038-005-MY2) and the Taipei Medical University for financially supporting this work.

\section{Author contributions}

JCK and DIK performed the experiment, DIK and TRK wrote the manuscript, JCK, DIK, S, FR, ES and TRK conceptualize, develop the methodology, and provided expertise and feedback.

\section{Declaration of interest}

The authors do not have any conflict of interest.

Received: 5 February 2022

Accepted: 1 March 2022

Published online: 1 March 2022

\section{References}

1. Yougbaré S, Chou H-L, Yang C-H, Krisnawati DI, Jazidie A, Nuh M, et al. Facet-dependent gold nanocrystals for effective photothermal killing of bacteria. J Hazard Mater. 2021;407: 124617. https://doi.org/10.1016/j. jhazmat.2020.124617

2. Li C-H, Kuo T-R, Su H-J, Lai W-Y, Yang P-C, Chen J-S, et al. Fluorescence-guided probes of aptamer-targeted gold nanoparticles with computed tomography imaging accesses for in vivo tumor resection. Sci Rep. 2015;5: 15675. https://doi.org/10.1038/srep15675

3. Mutalik C, Hsiao Y-C, Chang Y-H, Krisnawati DI, Alimansur M, Jazidie A, et al. High UV-VIS-NIR light-induced antibacterial activity by heterostructured $\mathrm{TiO}_{2}-\mathrm{FeS}_{2}$ nanocomposites. Int J Nanomedicine. 2020;15: 8911. https://doi.org/10.2147/IJN.S282689

4. Kuo T-R, Liao H-J, Chen Y-T, Wei C-Y, Chang C-C, Chen $\mathrm{Y}-\mathrm{C}$, et al. Extended visible to near-infrared harvesting of earth-abundant $\mathrm{FeS}_{2}-\mathrm{TiO}_{2}$ heterostructures for highly active photocatalytic hydrogen evolution. Green Chem. 2018;20: 1640-7. https://doi.org/10.1039/C7GC03173D

5. Zhu YP, Kuo TR, Li YH, Qi MY, Chen G, Wang JL, et al. Emerging dynamic structure of electrocatalysts unveiled by in situ X-ray diffraction/absorption spectroscopy. Energy Environ Sci. 2021;14: 1928-58. https://doi. org/10.1039/d0ee03903a

6. Tung CW, Kuo TR, Hsu CS, Chuang Y, Chen HC, Chang CK, et al. Light-Induced Activation of Adaptive Junction for Efficient Solar-Driven Oxygen Evolution: In Situ Unraveling the Interfacial Metal-Silicon Junction. Adv Energy Mater. 2019;9: 1901308. https://doi.org/10.1002/aenm.201901308

7. Yougbare S, Mutalik C, Krisnawati DI, Kristanto H, Jazidie A, Nuh M, et al. Nanomaterials for the Photothermal Killing of Bacteria. Nanomaterials. 2020;10: 1123. https:// doi.org/10.3390/nano10061123

8. Wang J, Tan HY, Kuo TR, Lin SC, Hsu CS, Zhu Y, et al. In situ identifying the dynamic structure behind activity of atomically dispersed platinum catalyst toward hydrogen evolution reaction. Small. 2021;17: 2005713. https:// doi.org/10.1002/smll.202005713

9. Yougbaré S, Mutalik C, Chung P-F, Krisnawati DI, Rinawati F, Irawan $\mathrm{H}$, et al. Gold nanorod-decorated metallic $\mathrm{MoS}_{2}$ nanosheets for synergistic photothermal and photodynamic antibacterial therapy. Nanomaterials. 2021;11: 3064. https://doi.org/10.3390/nano11113064 
10. Tan S-H, Yougbaré S, Tao H-Y, Chang C-C, Kuo T-R. Plasmonic gold nanoisland film for bacterial theranostics. Nanomaterials. 2021;11: 3139. https://doi.org/10.3390/ nano11113139

11. Mutalik C, Okoro G, Krisnawati DI, Jazidie A, Rahmawati EQ, Rahayu D, et al. Copper sulfide with morphologydependent photodynamic and photothermal antibacterial activities. J Colloid Interface Sci. 2022;607: 1825-35. https://doi.org/10.1016/j.jcis.2021.10.019

12. Yougbare S, Mutalik C, Okoro G, Lin IH, Krisnawati DI, Jazidie A, et al. Emerging Trends in Nanomaterials for Antibacterial Applications. Int J Nanomedicine. 2021;16: 5831-67. https://doi.org/10.2147/ijn.s328767

13. Mutalik C, Krisnawati DI, Patil SB, Khafid M, Atmojo DS, Santoso P, et al. Phase-dependent $\mathrm{MoS}_{2}$ nanoflowers for light-driven antibacterial application. ACS Sustain Chem Eng. 2021;9: 7904-12. https://doi.org/10.1021/ acssuschemeng.1c01868

14. Mutalik C, Wang DY, Krisnawati DI, Jazidie A, Yougbare S, Kuo TR. Light-Activated Heterostructured Nanomaterials for Antibacterial Applications. Nanomaterials. 2020;10: 643. https://doi.org/10.3390/nano10040643

15. Tan S-H, Yougbaré S, Chu H-L, Kuo T-R, Cheng T-M. Hemoglobin-Conjugated Gold Nanoclusters for Qualitative Analysis of Haptoglobin Phenotypes. Polymers. 2020;12: 2242. https://doi.org/10.3390/polym12102242

16. Du YX, Sheng HT, Astruc D, Zhu MZ. Atomically Precise Noble Metal Nanoclusters as Efficient Catalysts: A Bridge between Structure and Properties. Chem Rev. 2020;120: 526-622. https://doi.org/10.1021/acs.chemrev.8b00726

17. Kang X, Zhu MZ. Tailoring the photoluminescence of atomically precise nanoclusters. Chem Soc Rev. 2019;48: 2422-57. https://doi.org/10.1039/c8cs00800k

18. Kaur N, Aditya RN, Singh A, Kuo TR. Biomedical Applications for Gold Nanoclusters: Recent Developments and Future Perspectives. Nanoscale Res Lett. 2018;13: 302. https:// doi.org/10.1186/s11671-018-2725-9

19. Cheng TM, Chu HL, Lee YC, Wang DY, Chang CC, Chung $\mathrm{KL}$, et al. Quantitative Analysis of Glucose Metabolic Cleavage in Glucose Transporters Overexpressed Cancer Cells by Target-Specific Fluorescent Gold Nanoclusters. Anal Chem. 2018;90: 3974-80. https://doi.org/10.1021/ acs.analchem.7b04961

20. Krisnawati DI, Hsu P-H, Lin Y-H, Alimansur M, Atmojo DS, Rahmawati EQ et al. The Use of the ROS Scavenger Cysteine as a Surface Ligand of Metal Nanoclusters and Its Bactericidal Elimination Effect. Applied Sciences. 2021;11: 4095. https://doi.org/10.3390/app11094095

21. Hsu P-H, Yougbaré S, Kuo J-C, Krisnawati DI, Jazidie A, Nuh $\mathrm{M}$, et al. One-pot synthesis of thiol-modified liquid crystals conjugated fluorescent gold nanoclusters. Nanomaterials. 2020;10: 1755. https://doi.org/10.3390/nano10091755

22. Jia X, Li J, Han L, Ren J, Yang X, Wang E. DNA-hosted copper nanoclusters for fluorescent identification of single nucleotide polymorphisms. Acs Nano. 2012;6: 3311-7. https://doi.org/10.1021/nn3002455

23. Yougbare S, Chang T-K, Tan S-H, Kuo J-C, Hsu P-H, $\mathrm{Su} \mathrm{C}-\mathrm{Y}$, et al. Antimicrobial gold nanoclusters: recent developments and future perspectives. Int J Mol Sci. 2019;20: 2924. https://doi.org/10.3390/ijms20122924

24. Zhou M, Higaki T, Hu GX, Sfeir MY, Chen YX, Jiang DE, et al. Three-orders-of-magnitude variation of carrier lifetimes with crystal phase of gold nanoclusters. Science. 2019;364: 279-82. https://doi.org/10.1126/science.aaw8007

25. Chen TK, Lin HB, Cao YT, Yao QF, Xie JP. Interactions of Metal Nanoclusters with Light: Fundamentals and Applications. Adv Mater. 2103918. https://doi. org/10.1002/adma.202103918

26. Zheng K, Setyawati MI, Leong DT, Xie J. Antimicrobial gold nanoclusters. ACS nano. 2017;11: 6904-10. https:// doi.org/10.1021/acsnano.7b02035

27. Chang T-K, Cheng T-M, Chu H-L, Tan S-H, Kuo J-C, Hsu P-H, et al. Metabolic mechanism investigation of antibacterial active cysteine-conjugated gold nanoclusters in escherichia coli. ACS Sustain Chem Eng. 2019;7: 15479-86. https:// doi.org/10.1021/acssuschemeng.9b03048

28. Wang S, Wang Y, Peng Y, Yang X. Exploring the antibacteria performance of multicolor $\mathrm{Ag}$, $\mathrm{Au}$, and $\mathrm{Cu}$ nanoclusters. ACS Appl Mater Interfaces. 2019;11: 8461-9. https:// doi.org/10.1021/acsami.8b22143

29. Kuo J-C, Tan S-H, Hsiao Y-C, Mutalik C, Chen H-M, Yougbaré $S$, et al. Unveiling the Antibacterial Mechanism of Gold Nanoclusters via In Situ Transmission Electron Microscopy. ACS Sustain Chem Eng. 2021;10: 464-71. https://doi.org/10.1021/acssuschemeng.1c06714

30. Cheng T-M, Murad YM, Chang C-C, Yang M-C, Baral TN, Cowan A, et al. Single domain antibody against carcinoembryonic antigen-related cell adhesion molecule 6 (CEACAM6) inhibits proliferation, migration, invasion and angiogenesis of pancreatic cancer cells. European Journal of Cancer. 2014;50: 713-21. https://doi.org/10.1016/j.ejca.2012.07.019

31. Shang L, Dong S, Nienhaus GU. Ultra-small fluorescent metal nanoclusters: synthesis and biological applications. Nano Today. 2011;6: 401-18. https://doi.org/10.1016/j. nantod.2011.06.004

32. Luo Z, Yuan X, Yu Y, Zhang Q Leong DT, Lee JY, et al. From Aggregation-Induced Emission of $\mathrm{Au}(\mathrm{I})-\mathrm{Thiolate}$ Complexes to Ultrabright $\mathrm{Au}(0) @ \mathrm{Au}(\mathrm{I})-\mathrm{Thiolate}$ CoreShell Nanoclusters. J Am Chem Soc. 2012;134: 16662-70. https://doi.org/10.1021/ja306199p

33. Goswami N, Yao QF, Luo ZT, Li JG, Chen TK, Xie JP. Luminescent Metal Nanoclusters with AggregationInduced Emission. J Phys Chem Lett. 2016;7: 962-75. https://doi.org/10.1021/acs.jpclett.5b02765

34. Bera D, Goswami N. Driving Forces and Routes for Aggregation-Induced Emission-Based Highly Luminescent Metal Nanocluster Assembly. J Phys Chem Lett. 2021;12: 9033-46. https://doi.org/10.1021/acs.jpclett.1c02406 